\title{
WEAK CONVERGENCE LIMITS FOR CLOSED CYCLIC NETWORKS OF QUEUES WITH MULTIPLE BOTTLENECK NODES
}

\author{
OLE STENZEL* AND \\ HANS DADUNA, ${ }^{* *}$ University of Hamburg
}

\begin{abstract}
We consider a sequence of cycles of exponential single-server nodes, where the number of nodes is fixed and the number of customers grows unboundedly. We prove a central limit theorem for the cycle time distribution. We investigate the idle time structure of the bottleneck nodes and the joint sojourn time distribution that a test customer observes at the nonbottleneck nodes during a cycle. Furthermore, we study the filling behaviour of the bottleneck nodes, and show that the single bottleneck and multiple bottleneck cases lead to different asymptotic behaviours.
\end{abstract}

Keywords: Cyclic exponential network; cycle time; idle time; filling behaviour; bottleneck structure; central limit theorem

2010 Mathematics Subject Classification: Primary 60K25

\section{Introduction}

In this paper we analyze the behaviour of cyclic single-server Gordon-Newell networks in equilibrium as the number of customers in the system increases to $\infty$. There are two different cases to consider.

Case (i): All nodes have the same service rate. In this case the customers are uniformly distributed over the nodes.

Case (ii): Different service rates exist. In this case at least one bottleneck (the node with the smallest service rate) exists. Then almost all the customers will be queued up at the bottleneck nodes.

In this paper we focus on case (ii). We investigate a customer's cycle time distribution for which a central limit theorem will be proved. Furthermore, we characterize the differences in the filling behaviour of nodes between the single bottleneck and the multiple bottleneck cases. An important aspect of the networks' behaviour is the mean idle time of the bottleneck nodes during the cycle of a test customer.

The paper is organized as follows. In Section 2 the closed cyclic network of queues is described and the properties needed in the sequel are referenced. In Section 3 we investigate customers' cycle time distribution and discuss its connection to the mean idle times of bottlenecks. This connection stems from the description of cycle times from the viewpoint of a node.

Received 28 January 2010; revision received 21 September 2011.

* Current address: Institute of Stochastics, Ulm University, Helmholtzstrasse 18, 89069 Ulm, Germany.

** Postal address: Center of Mathematical Statistics and Stochastic Processes, University of Hamburg, Bundesstrasse 55, 20146 Hamburg, Germany. Email address: daduna@math.uni-hamburg.de 
For a population size of $N$, the time between $N$ successive departures from a specified node is the cycle time of the first of the departing customers [3].

Starting with the limiting cycle time distribution, we prove a central limit theorem for $N \rightarrow \infty$, which generalizes the central limit theorem for the cycle time when all service rates are distinct [10]. In Section 4 we analyze the differences in the filling behaviour of the network between the single bottleneck and multiple bottleneck cases. There is a significant difference with respect to the mean idle times between these two cases. Considering the admissible service rate vectors of the cycles as parameter space, we observe a jump in the mean idle times, which constitutes a phase transition, when reaching a certain boundary region of the parameter space. Motivated by this, we analyze in detail the speed with which bottleneck nodes are filled up and find a fundamental difference in the speed of convergence between the single and multiple bottleneck cases.

In Section 5 we prove a weak convergence theorem for the joint steady-state queue length distribution and the joint sojourn times of a test customer at the nonbottleneck nodes. Section 6 is devoted to the cycle time in homogeneous cycles, i.e. all nodes have the same service rate. A central limit theorem is directly obtained. In Section 7 we discuss the problems arising with multiple servers in the system.

\section{Model specification and previous results}

We consider a closed cycle consisting of $M$ nodes $Q[1], \ldots, Q[M]$ and $N$ indistinguishable customers. Node $Q[i], i=1, \ldots, M$, is a single server with infinite waiting room, under the FCFS (first-come-first-served) regime. The dynamic of the system is as follows. If a customer arrives at node $Q[i], i=1, \ldots, M$, and finds an idle service channel, her service begins immediately. Otherwise, she will join the tail of the queue. Service times at node $Q[i]$ are $\exp \left(\mu_{i}\right)$ distributed. If the service of the customer at node $Q[i]$ is complete, she moves immediately to node $Q[i+1]$ (with $Q[M+1]:=Q[1]$ ). The other customers waiting in the line (if there are any) move one position forward. All movements of customers happen without any time lag. Service times at node $Q[i]$ form a sequence of independent and identically distributed (i.i.d.) random variables, independent of the service times at other nodes.

Denote by $X_{i}^{(M, N)}(t)$ the queue length at node $Q[i]$, i.e. the number of customers present at node $Q[i]$ (waiting or in service), at time $t \geq 0$. Define

$$
X^{(M, N)}(t):=\left(X_{1}^{(M, N)}(t), \ldots, X_{M}^{(M, N)}(t)\right)
$$

to be the joint queue length vector at time $t$ and $X^{(M, N)}:=\left(X^{(M, N)}(t), t \geq 0\right)$ to be the joint queue length process. The state space of $X^{(M, N)}$ is

$$
Z(M, N):=\left\{\left(n_{1}, \ldots, n_{M}\right) \in \mathbb{N}^{M} \mid n_{1}+\cdots+n_{M}=N\right\} .
$$

Proposition 2.1. ([11].) The joint queue length process $X^{(M, N)}=\left(X^{(M, N)}(t), t \geq 0\right)$ is a strong Markov process, which is irreducible and positive recurrent. The limiting and stationary distribution is

$$
\pi^{(M, N)}(n)=G(M, N)^{-1} \prod_{i=1}^{M}\left(\frac{1}{\mu_{i}}\right)^{n_{i}}
$$

with $n=\left(n_{1}, \ldots, n_{M}\right) \in Z(M, N)$ and normalising constant

$$
G(M, N)=\sum_{n \in Z(M, N)} \prod_{i=1}^{M}\left(\frac{1}{\mu_{i}}\right)^{n_{i}} .
$$


In the following we assume that the joint queue length process is in equilibrium. We therefore omit the time parameter $t$, i.e. we write $X^{(M, N)}$ instead of $X^{(M, N)}(t)$.

We investigate the individual behaviour of the customers in the cycle. Therefore, we fix on one customer, which we call the test customer (TC). The random time between two entrances of the TC into node $Q[1]$ is called the cycle time. We obtain a sequence of cycle times $\left(c_{N}^{(i)}\right.$, $i \in \mathbb{N}_{+}$), where $c_{N}^{(i)}$ is the $i$ th passage time through the cycle.

Theorem 2.1. (Limiting distribution of the cycle time and sojourn time vector [4].) The limiting distribution of the TC's cycle time is given by its Laplace-Stieltjes transform (LST)

$$
\psi^{(M, N)}(\theta)=\sum_{n \in Z(M, N-1)} \pi^{(M, N-1)}(n) \prod_{j=1}^{M}\left(\frac{\mu_{j}}{\mu_{j}+\theta}\right)^{n_{j}+1}, \quad \theta \geq 0,
$$

where $\pi^{(M, N-1)}\left(n_{1}, \ldots, n_{M}\right)$ (defined in (2.1)) is the steady-state probability that at the arrival instants of the TC at node $Q[1]$, he observes $n_{1}$ customers before him at node $Q[1]$ and $n_{i}$ customers present at node $Q[i], i=2, \ldots, M$, without counting himself.

The limiting joint distribution of the TC's successive sojourn times during a cycle is given by its $L S T$

$$
\begin{aligned}
\phi^{(M, N)}\left(\theta_{1}, \ldots, \theta_{M}\right)= & \sum_{n \in Z(M, N-1)} \pi^{(M, N-1)}(n) \\
& \times \prod_{j=1}^{M}\left(\frac{\mu_{j}}{\mu_{j}+\theta_{j}}\right)^{n_{j}+1}, \quad \theta_{j} \geq 0, j=1, \ldots, M .
\end{aligned}
$$

To investigate in more detail the cycle time distribution with $\operatorname{LST} \psi^{(M, N)}(\theta)$, Harrison [13] obtained by direct (but tedious) inversion explicit expressions for the density functions of distributions with LST

$$
\prod_{j=1}^{M}\left(\frac{\mu_{j}}{\mu_{j}+\theta}\right)^{n_{j}+1}, \quad \theta \geq 0
$$

see [13, Theorem 1]. Applying the techniques we will develop in the present paper to the mixture of densities with LST (2.2) may lead to a proof of a central limit theorem without transform methods.

If we define the cycle time for the TC to be the time between two successive entrances of the TC into some node $Q[i]$, in the case of all service times being exponential, the cycle time distribution would be the same; for details, see [9]. If nonexponential service times occur, this property does not hold; see, e.g. [2, Section 4].

Notation. Denote by $c_{N}$ a random variable with LST (2.2), i.e. $c_{N} \sim \psi^{(M, N)}$. Whenever we refer to a cycle time, we mean a random variable $c_{N}$ with $\operatorname{LST} \psi^{(M, N)}$. In any case the number, $M$, of nodes is fixed. Similarly, a vector with nonnegative coordinates $\left(S_{1}^{(N)}, S_{2}^{(N)}, \ldots, S_{M}^{(N)}\right) \sim$ $\phi^{(M, N)}\left(\theta_{1}, \ldots, \theta_{M}\right)$ is considered as the vector of the TC's successive sojourn times during a cycle under customer stationary conditions.

Definition 2.1. The node $Q[i], i=1, \ldots, M$, is a bottleneck node if $\mu_{i}=\min \left\{\mu_{k}, k=\right.$ $1, \ldots, M\}$.

To simplyfy the calculations, we assume without loss of generality that $\mu_{1} \leq \cdots \leq \mu_{M}$.

Notation. Denote by $m$ the number of different service rates, i.e. the number of different values of $\mu_{1} \leq \cdots \leq \mu_{M}$. The distinct service rates will be denoted by $\eta_{1}<\eta_{2}<\cdots<\eta_{m}$. 
Let $v_{l}, 1 \leq l \leq m$, be the number of $i \in\{1, \ldots, M\}$ with $\mu_{i}=\eta_{l}$. So $v_{1}$ is the number of bottlenecks, and $\mu_{1}=\eta_{1}$ and $\mu_{M}=\eta_{m}$.

Gordon and Newell [11] proved that the bottlenecks have an overwhelming influence on the steady-state behaviour of a closed network, whenever the number, $M$, of nodes is constant and $N \rightarrow \infty$ :

$$
\begin{gathered}
\lim _{N \rightarrow \infty} \mathrm{P}\left(X_{1}^{(M, N)} \geq n\right)=1 \quad \text { for all } n \in \mathbb{N} \\
\lim _{N \rightarrow \infty} \mathrm{P}\left(X_{2}^{(M, N)}=n_{2}, \ldots, X_{M}^{(M, N)}=n_{M}\right)=\prod_{j=2}^{M}\left(1-\frac{\mu_{1}}{\mu_{j}}\right)\left(\frac{\mu_{1}}{\mu_{j}}\right)^{n_{j}}
\end{gathered}
$$

for all $n_{j} \geq 0, j=2, \ldots, M$. The usual interpretation for a cyclic network is that, with an increasing number of customers, the bottleneck approaches a Poissonian source feeding the rest of the network, while all the other nodes eventually form an open ergodic tandem system, the behaviour of which is well understood: local geometrical queue length distribution and independence over the nodes in steady state. A similar property holds for sojourn times and partial cycle times; see Theorem 5.1 of [10].

A similar interpretation should hold if several bottlenecks occur which divide the cycle into bottlenecks and (possibly empty) sequences of nonbottleneck nodes between them. This will be examined in Section 5 .

If $Q[1]$ is the only bottleneck $\left(\mu_{1}<\mu_{i}\right.$ for all $\left.i \neq 2\right)$, it is not surprising to the TC that, for large populations, almost all other customers are waiting before her at $Q[1]$ when her cycle commences. It follows (see [3]) that, as $N \rightarrow \infty$,

$$
\mathrm{E}\left[c_{N}\right]=N \mu_{1}^{-1}\left\{1+O\left(\left[\frac{\mu_{1}}{\mu_{2}}\right]^{N}\right)\right\}, \quad \operatorname{var}\left(c_{N}\right)=N \mu_{1}^{-2}\left\{1+O\left(\left[\frac{\mu_{1}}{\mu_{2}}\right]^{N}\right)\right\} .
$$

From (2.4), in heavy traffic the slowest queue generates the main fraction of the TC's cycle time. This suggests approximating the cycle time distribution for large $N$ by the sum of $N$ consecutive service times at the slowest queue. This is supported by Chow's [7] observation that in a two-stage cycle a result parallel to (2.4) holds for the LST of the cycle times as well. This suggests that a central limit theorem for the rescaled cycle time holds, when the population size tends to $\infty$, while the number of stations remains fixed.

The central limit theorem for the rescaled cycle time was proved in [10, Theorem 4.1] for pairwise distinct service rates, i.e. in the case of a single bottleneck. Furthermore, weak convergence of the joint sojourn time vectors is proved, with the bottleneck under central limit scaling, and the nonbottlenecks without scaling.

Moreover, in the general setting the usual interpretation suggests that the unscaled sojourn times at the nonbottleneck nodes should converge to exponential distributions. This will be proved in Theorem 5.1.

\section{Cycle times and idle times of bottlenecks}

The cycle time is defined as the time experienced by the TC between two successive entrances into $Q[1]$. We now adopt the following alternative description of cycle times due to Boxma [3, p. 19], which is defined from the viewpoint of a server. We start by observing server $Q[1]$ the instant the TC leaves $Q$ [1]. Denote by $i_{1}$ the idle time (which may be 0 ) until the next customer starts his service at node $Q[1]$. Then, denote by $\tau_{1}$ the service time of the next customer. When this customer leaves $Q[1]$, denote by $i_{2}$ the next idle time (which may again be 0 ) of 
the service channel, and so on. By and by, customers pass $Q[1]$, and we denote the idle times by $i_{j}$ (being 0 if customers are waiting at $Q[1]$ ) and the service times by $\tau_{j}, j=1, \ldots, N$. After the TC leaves $Q[1]$ again, we obtain the cycle time as the sum of the service times and idle times. With $\delta_{N}:=\tau_{1}+\cdots+\tau_{N}$, the sum of $N$ i.i.d. $\exp \left(\mu_{1}\right)$-distributed service times, and $\rho_{N}:=i_{1}+\cdots+i_{N}$, the cumulative idle time, we have

$$
c_{N}=\left(i_{1}+\cdots+i_{N}\right)+\left(\tau_{1}+\cdots+\tau_{N}\right)=\rho_{N}+\delta_{N} .
$$

A first simple observation yields a bound for $\mathrm{E}\left[\rho_{N}\right]$ which is independent on $N$. We have

$$
\begin{aligned}
\mathrm{E}\left[c_{N}\right] & \stackrel{(2.3)}{=} \sum_{n \in Z(M, N-1)} \pi^{(M, N-1)}(n) \sum_{j=1}^{M}\left(n_{j}+1\right) \underbrace{\mu_{j}^{-1}}_{\leq \mu_{1}^{-1}} \\
& \leq \sum_{n \in Z(M, N-1)} \pi^{(M, N-1)}(n) \mu_{1}^{-1} \underbrace{\sum_{j=1}^{M}\left(n_{j}+1\right)}_{=N+M-1} \\
& =(N+M-1) \mu_{1}^{-1} \sum_{n \in Z(M, N-1)} \pi^{(M, N-1)}(n) \\
& =(N+M-1) \mu_{1}^{-1} .
\end{aligned}
$$

Since $\mathrm{E}\left[\rho_{N}\right]=\mathrm{E}\left[c_{N}\right]-\mathrm{E}\left[\delta_{N}\right]=\mathrm{E}\left[c_{N}\right]-N \mu_{1}^{-1}$, it follows that

$$
\mathrm{E}\left[\rho_{N}\right] \leq(M-1) \mu_{1}^{-1} \text {. }
$$

Boxma [3] observed that, for exactly one bottleneck in the cycle, we have $\mathrm{E}\left[\rho_{N}\right] \rightarrow 0$ as $N \rightarrow \infty$. Equation (3.1) splits the cycle time into $\delta_{N}$, with $\mathrm{E}\left[\delta_{N}\right] \rightarrow \infty$ and $\rho_{N}$, with bounded mean values; see (3.2). So, for large $N$, the influence of $\rho_{N}$ on the cycle time should not be significant compared to that of $\delta_{N}$ :

$$
\frac{c_{N}-\mathrm{E}\left[c_{N}\right]}{\sqrt{\operatorname{var}\left(c_{N}\right)}} \approx \frac{\delta_{N}-\mathrm{E}\left[\delta_{N}\right]}{\sqrt{\operatorname{var}\left(\delta_{N}\right)}} .
$$

For the sequence of standardized random variables (indexed by $N$ ) on the right-hand side of the above expression, a central limit theorem holds. The main problem is to sharpen and extend (3.2).

\subsection{Idle times at the bottlenecks}

Recall that node $Q[1]$ is always a bottleneck and that $v_{1} \geq 1$ is the number of bottlenecks. For the expected cumulative idle times at $Q[1]$ during the TC's cycle in the steady-state regime, we have a precise asymptotic. This is obtained from moment properties of the cycle time, which will be used to prove the central limit theorem.

Theorem 3.1. It holds that

$$
\lim _{N \rightarrow \infty}\left(\mathrm{E}\left[c_{N}\right]-\mu_{1}^{-1} N\right)=\mu_{1}^{-1}\left(v_{1}-1\right) .
$$

Theorem 3.2. It holds that

$$
\lim _{N \rightarrow \infty}\left(\operatorname{var}\left(c_{N}\right)-\mu_{1}^{-2} N\right)=\mu_{1}^{-2}\left(v_{1}-1\right) .
$$


An immediate corollary is as follows.

Corollary 3.1. It holds that

$$
\lim _{N \rightarrow \infty} \mathrm{E}\left[\rho_{N}\right]=\mu_{1}^{-1}\left(v_{1}-1\right)
$$

and

$$
\lim _{N \rightarrow \infty}\left(\operatorname{var}\left(\rho_{N}\right)+\operatorname{cov}\left(\rho_{N}, \delta_{N}\right)\right)=\mu_{1}^{-2}\left(v_{1}-1\right) .
$$

While (3.6) does not admit a direct interpretation, (3.5) has the following appealing interpretation. Assume that we have a single bottleneck, $Q$ [1]. Then, from (3.2), it follows that $\lim _{N \rightarrow \infty} \mathrm{E}\left[\rho_{N}\right]=0$. Assume further that, for $Q[2], Q[3], \ldots, Q[k]$, with $k<M$, we let $\mu_{j} \rightarrow \mu_{1}, j=2, \ldots, k$ converge. Then we have a discontinuity of the asymptotic expected idle times in this limiting procedure.

Alternatively, we could consider the sequence of networks to be dependent on the parameter vector $\left(\mu_{1}, \ldots, \mu_{M}\right)$, and assume that the parameters vary in such a way that we start with $Q[1]$ as the only bottleneck. Consider the function which maps any sequence of networks to the value $\lim _{N \rightarrow \infty} \mathrm{E}\left[\rho_{N}\right]$. This function starts at 0 and stays there until at least one other $\mu_{j}$ reaches $\mu_{j}=\mu_{1}$. Then the mean value function $\mathrm{E}\left[\rho_{N}\right]$ immediately jumps to a value greater than or equal to $\mu_{1}^{-1}>0$ and a phase transition occurs when the parameter vector reaches the boundary region defined by $\left\{\mu_{1}=\mu_{2}\right\}$. We shall discuss this behaviour in more detail in Section 4.

The proofs of Theorems 3.1 and 3.2 need several preparatory steps which will be given now. The following interchange formula for sums and products is a consequence of Harrison's formula [12].

Lemma 3.1. We have

$$
\begin{array}{r}
\sum_{n \in Z(M, N)} \prod_{j=1}^{M}\left(\frac{1}{\mu_{j}}\right)^{n_{j}}=\sum_{l=1}^{m} \sum_{a \in Z\left(m, \nu_{l}-1\right)}(-1)^{a_{l}-v_{l}+1}\left(\frac{1}{\eta_{l}}\right)^{N-v_{l}+a_{l}+1}\left(\begin{array}{c}
N+a_{l} \\
N
\end{array}\right) \\
\times\left(\prod_{u=1, u \neq l}^{m} \eta_{u}^{v_{u}}\left(\begin{array}{c}
v_{u}+a_{u}-1 \\
v_{u}-1
\end{array}\right)\left(\frac{1}{\eta_{u}-\eta_{l}}\right)^{v_{u}+a_{u}}\right) .
\end{array}
$$

Proof. We have

$$
\begin{array}{r}
\sum_{n \in Z(M, N)} \prod_{j=1}^{M}\left(\frac{1}{\mu_{j}}\right)^{n_{j}} \\
\stackrel{(*)}{=} \sum_{l=1}^{m} \sum_{a \in Z\left(m, v_{l}-1\right)}(-1)^{a_{l}-v_{l}+1}\left(\frac{1}{\eta_{l}}\right)^{N+M-v_{l}}\left(\begin{array}{c}
N+a_{l} \\
N
\end{array}\right) \\
\times\left(\prod_{u=1, u \neq l}^{m} \eta_{u}^{-a_{u}}\left(\begin{array}{c}
v_{u}+a_{u}-1 \\
v_{u}-1
\end{array}\right)\left(\frac{1}{\eta_{l}}-\frac{1}{\eta_{u}}\right)^{-v_{u}-a_{u}}\right) \\
=\sum_{l=1}^{m} \sum_{a \in Z\left(m, v_{l}-1\right)}(-1)^{a_{l}-v_{l}+1}\left(\frac{1}{\eta_{l}}\right)^{N+M-v_{l}}\left(\begin{array}{c}
N+a_{l} \\
N
\end{array}\right) \\
\times\left(\prod_{u=1, u \neq l}^{m} \eta_{u}^{-a_{u}}\left(\begin{array}{c}
v_{u}+a_{u}-1 \\
v_{u}-1
\end{array}\right)\left(\frac{\eta_{l} \eta_{u}}{\eta_{u}-\eta_{l}}\right)^{v_{u}+a_{u}}\right)
\end{array}
$$




$$
\begin{array}{r}
=\sum_{l=1}^{m} \sum_{a \in Z\left(m, v_{l}-1\right)}(-1)^{a_{l}-v_{l}+1}\left(\frac{1}{\eta_{l}}\right)^{N+M-v_{l}}\left(\begin{array}{c}
N+a_{l} \\
N
\end{array}\right) \eta_{l}^{\sum_{u \neq l}\left(v_{u}+a_{u}\right)} \\
\times\left(\prod_{u=1, u \neq l}^{m} \eta_{u}^{v_{u}}\left(\begin{array}{c}
v_{u}+a_{u}-1 \\
v_{u}-1
\end{array}\right)\left(\frac{1}{\eta_{u}-\eta_{l}}\right)^{v_{u}+a_{u}}\right) \\
=\sum_{l=1}^{m} \sum_{a \in Z\left(m, v_{l}-1\right)}(-1)^{a_{l}-v_{l}+1}\left(\frac{1}{\eta_{l}}\right)^{N-v_{l}+a_{l}+1}\left(\begin{array}{c}
N+a_{l} \\
N
\end{array}\right) \\
\times\left(\prod_{u=1, u \neq l}^{m} \eta_{u}^{v_{u}}\left(\begin{array}{c}
v_{u}+a_{u}-1 \\
v_{u}-1
\end{array}\right)\left(\frac{1}{\eta_{u}-\eta_{l}}\right)^{v_{u}+a_{u}}\right)
\end{array}
$$

where $\stackrel{(*)}{=}$, is the generalization of Harrison's formula; see [18, Proposition 1.32]. Note that the expression therein contains typos; see [15, Equation (1.19)].

Definition 3.1. For the general cycle, the following constants will help to keep some computations shorter:

(i) $K_{1}:=\mathbf{1}_{\left\{v_{1}>1\right\}} \sum_{u=2}^{m} \frac{v_{u}}{\eta_{u}-\eta_{1}}$,

(ii) $K_{2}:=\mathbf{1}_{\left\{v_{1}>2\right\}} \sum_{a_{2}+\cdots+a_{M}=2} \prod_{u=2}^{m}\left(\frac{1}{\eta_{u}-\eta_{1}}\right)^{a_{u}}\left(\begin{array}{c}v_{u}+a_{u}-1 \\ v_{u}-1\end{array}\right)$,

(iii) $\tilde{K}_{1}:=\left(v_{1}-1\right) K_{1}$,

(iv) $\tilde{K}_{2}:=\left(v_{1}-1\right)\left(v_{1}-2\right) K_{2}$,

(v) by $\left(a_{N}\right)_{N \in \mathbb{N}}$ we denote sequences with the property

$$
\begin{aligned}
a_{N}:= & 1-\eta_{1} \frac{1}{N+v_{1}-1} \tilde{K}_{1}+\eta_{1}^{2} \frac{1}{\left(N+v_{1}-1\right)\left(N+v_{1}-2\right)} \tilde{K}_{2} \\
& +O\left(\left(\frac{1}{N}\right)^{3}\right) .
\end{aligned}
$$

Note that $K_{1}=\tilde{K}_{1}=0$ if $v_{1}=1$ and that $K_{2}=\tilde{K}_{2}=0$ if $v_{1} \leq 2$.

Lemma 3.2. Let $\left(a_{N}\right)_{N \in \mathbb{N}}$ denote a sequence with property (3.8). Then

$$
\lim _{N \rightarrow \infty} N\left(a_{N}-1\right)=-\eta_{1} \tilde{K}_{1}
$$

Proposition 3.1. The normalization constant in the general cycle is

$$
\begin{aligned}
G(M, N)=\sum_{l=1}^{m} \sum_{a \in Z\left(m, v_{l}-1\right)}(-1)^{a_{l}-v_{l}+1}\left(\frac{1}{\eta_{l}}\right)^{N-v_{l}+a_{l}+1}\left(\begin{array}{c}
N+a_{l} \\
N
\end{array}\right) \\
\times\left(\prod_{u=1, u \neq l}^{m} \eta_{u}^{v_{u}}\left(\begin{array}{c}
v_{u}+a_{u}-1 \\
v_{u}-1
\end{array}\right)\left(\frac{1}{\eta_{u}-\eta_{l}}\right)^{v_{u}+a_{u}}\right) .
\end{aligned}
$$


The normalization constants obey the asymptotic expansion

$$
G(M, N)=\left(\frac{1}{\eta_{1}}\right)^{N}\left(\begin{array}{c}
N+v_{1}-1 \\
N
\end{array}\right)\left(\prod_{u=2}^{m}\left(\frac{\eta_{u}}{\eta_{u}-\eta_{1}}\right)^{v_{u}}\right) a_{N},
$$

where $\left(a_{N}\right)_{N \in \mathbb{N}}$ is the sequence given in Definition 3.1(v).

Equation (3.11) tells us that the partition function $G(M, N)$ is dominated by the term

$$
\left(\frac{1}{\eta_{1}}\right)^{N}\left(\begin{array}{c}
N+v_{1}-1 \\
N
\end{array}\right) \prod_{u=2}^{m}\left(\frac{\eta_{u}}{\eta_{u}-\eta_{1}}\right)^{v_{u}} .
$$

The proof of (3.11) is of a prototype structure for many of our later arguments. We therefore give the details.

Proof of Proposition 3.1. Equation (3.10) follows immediately from (3.7).

We start with the normalization constant in the form (3.10) and split the term where $l=1$, i.e. the summand representing the bottlenecks. As will be seen later, only this summand is persistent if $N \rightarrow \infty$. We have

$$
\begin{aligned}
& G(M, N)=\sum_{l=2}^{m} \sum_{a \in Z\left(m, v_{l}-1\right)}(-1)^{a_{l}-v_{l}+1}\left(\frac{1}{\eta_{l}}\right)^{N-v_{l}+a_{l}+1}\left(\begin{array}{c}
N+a_{l} \\
N
\end{array}\right) \\
& \times\left(\prod_{u=1, u \neq l}^{m} \eta_{u}^{v_{u}}\left(\begin{array}{c}
v_{u}+a_{u}-1 \\
v_{u}-1
\end{array}\right)\left(\frac{1}{\eta_{u}-\eta_{l}}\right)^{v_{u}+a_{u}}\right) \\
& +\sum_{a \in Z\left(m, v_{1}-1\right)}(-1)^{a_{1}-v_{1}+1}\left(\frac{1}{\eta_{1}}\right)^{N-v_{1}+a_{1}+1}\left(\begin{array}{c}
N+a_{1} \\
N
\end{array}\right) \\
& \times\left(\prod_{u=2}^{m} \eta_{u}^{v_{u}}\left(\begin{array}{c}
v_{u}+a_{u}-1 \\
v_{u}-1
\end{array}\right)\left(\frac{1}{\eta_{u}-\eta_{1}}\right)^{v_{u}+a_{u}}\right) \\
& =\sum_{l=2}^{m} \sum_{a \in Z\left(m, v_{l}-1\right)}(-1)^{a_{l}-v_{l}+1}\left(\frac{1}{\eta_{l}}\right)^{N-v_{l}+a_{l}+1}\left(\begin{array}{c}
N+a_{l} \\
N
\end{array}\right) \\
& \times\left(\prod_{u=1, u \neq l}^{m} \eta_{u}^{v_{u}}\left(\begin{array}{c}
v_{u}+a_{u}-1 \\
v_{u}-1
\end{array}\right)\left(\frac{1}{\eta_{u}-\eta_{l}}\right)^{v_{u}+a_{u}}\right) \\
& +\sum_{a \in Z\left(m, v_{1}-1\right), a_{1}<v_{1}-3}(-1)^{a_{1}-v_{1}+1}\left(\frac{1}{\eta_{1}}\right)^{N-v_{1}+a_{1}+1}\left(\begin{array}{c}
N+a_{1} \\
N
\end{array}\right) \\
& \times\left(\prod_{u=2}^{m} \eta_{u}^{v_{u}}\left(\begin{array}{c}
v_{u}+a_{u}-1 \\
v_{u}-1
\end{array}\right)\left(\frac{1}{\eta_{u}-\eta_{1}}\right)^{v_{u}+a_{u}}\right) \\
& +\sum_{a \in Z\left(m, v_{1}-1\right), a_{1} \geq v_{1}-3}(-1)^{a_{1}-v_{1}+1}\left(\frac{1}{\eta_{1}}\right)^{N-v_{1}+a_{1}+1}\left(\begin{array}{c}
N+a_{1} \\
N
\end{array}\right) \\
& \times\left(\prod_{u=2}^{m} \eta_{u}^{v_{u}}\left(\begin{array}{c}
v_{u}+a_{u}-1 \\
v_{u}-1
\end{array}\right)\left(\frac{1}{\eta_{u}-\eta_{1}}\right)^{v_{u}+a_{u}}\right) .
\end{aligned}
$$


Splitting the last expressions for $a_{1} \geq v_{1}-3$, i.e. $a_{1}=v_{1}-1, a_{1}=v_{1}-2$, and $a_{1}=v_{1}-3$, yields

$$
\begin{aligned}
& G(M, N)=\sum_{l=2}^{m} \sum_{a \in Z\left(m, v_{l}-1\right)}(-1)^{a_{l}-v_{l}+1}\left(\frac{1}{\eta_{l}}\right)^{N-v_{l}+a_{l}+1}\left(\begin{array}{c}
N+a_{l} \\
N
\end{array}\right) \\
& \times\left(\prod_{u=1, u \neq l}^{m} \eta_{u}^{v_{u}}\left(\begin{array}{c}
v_{u}+a_{u}-1 \\
v_{u}-1
\end{array}\right)\left(\frac{1}{\eta_{u}-\eta_{l}}\right)^{v_{u}+a_{u}}\right) \\
& +\sum_{a \in Z\left(m, \nu_{1}-1\right), a_{1}<v_{1}-3}(-1)^{a_{1}-v_{1}+1}\left(\frac{1}{\eta_{1}}\right)^{N-v_{1}+a_{1}+1}\left(\begin{array}{c}
N+a_{1} \\
N
\end{array}\right) \\
& \times\left(\prod_{u=2}^{m} \eta_{u}^{v_{u}}\left(\begin{array}{c}
v_{u}+a_{u}-1 \\
v_{u}-1
\end{array}\right)\left(\frac{1}{\eta_{u}-\eta_{1}}\right)^{v_{u}+a_{u}}\right) \\
& +\left(\frac{1}{\eta_{1}}\right)^{N-2}\left(\begin{array}{c}
N+v_{1}-3 \\
N
\end{array}\right)\left(\prod_{u=2}^{m}\left(\frac{\eta_{u}}{\eta_{u}-\eta_{1}}\right)^{v_{u}}\right) K_{2} \\
& -\left(\frac{1}{\eta_{1}}\right)^{N-1}\left(\begin{array}{c}
N+v_{1}-2 \\
N
\end{array}\right)\left(\prod_{u=2}^{m}\left(\frac{\eta_{u}}{\eta_{u}-\eta_{1}}\right)^{v_{u}}\right) K_{1} \\
& +\left(\frac{1}{\eta_{1}}\right)^{N}\left(\begin{array}{c}
N+v_{1}-1 \\
N
\end{array}\right) \prod_{u=2}^{m}\left(\frac{\eta_{u}}{\eta_{u}-\eta_{1}}\right)^{v_{u}} .
\end{aligned}
$$

A short comment may be in order here. So far, we have only split the sum. First, we extracted the summand with $l=1$, then we extracted the summands with $a_{1} \geq v_{1}-3$. Finally, we wrote down the explicit expressions for $a_{1}=v_{1}-1, a_{1}=v_{1}-2$, and $a_{1}=v_{1}-3$. Now we factor out

$$
\left(\frac{1}{\eta_{1}}\right)^{N}\left(\begin{array}{c}
N+v_{1}-1 \\
N
\end{array}\right) \prod_{u=2}^{m}\left(\frac{\eta_{u}}{\eta_{u}-\eta_{1}}\right)^{v_{u}} .
$$

Since

$$
\lim _{N \rightarrow \infty} N^{p}\left(\frac{\eta_{1}}{\eta_{l}}\right)^{N}=0, \quad p \in \mathbb{N}, 2 \leq l \leq m,
$$

it follows that

$$
\begin{aligned}
& \left(\left(\frac{1}{\eta_{1}}\right)^{N}\left(\begin{array}{c}
N+v_{1}-1 \\
N
\end{array}\right) \prod_{u=2}^{m}\left(\frac{\eta_{u}}{\eta_{u}-\eta_{1}}\right)^{v_{u}}\right)^{-1} \\
& \times \sum_{l=2}^{m} \sum_{a \in Z\left(m, v_{l}-1\right)}(-1)^{a_{l}-v_{l}+1}\left(\frac{1}{\eta_{l}}\right)^{N-v_{l}+a_{l}+1}\left(\begin{array}{c}
N+a_{l} \\
N
\end{array}\right) \\
& \times\left(\prod_{u=1, u \neq l}^{m} \eta_{u}^{v_{u}}\left(\begin{array}{c}
v_{u}+a_{u}-1 \\
v_{u}-1
\end{array}\right)\left(\frac{1}{\eta_{u}-\eta_{l}}\right)^{v_{u}+a_{u}}\right) \\
& =O\left(\left(\frac{1}{N}\right)^{r}\right) \text { for all } r \in \mathbb{N} .
\end{aligned}
$$


We choose $r=3$, yielding $O\left((1 / N)^{3}\right)$. Since

$$
\left(\begin{array}{c}
N+a_{1} \\
N
\end{array}\right) /\left(\begin{array}{c}
N+v_{1}-1 \\
N
\end{array}\right)=O\left(\left(\frac{1}{N}\right)^{3}\right) \quad \text { for } a_{1}<v_{1}-3
$$

it follows that

$$
\begin{aligned}
G(M, N)= & \left(\frac{1}{\eta_{1}}\right)^{N}\left(\begin{array}{c}
N+v_{1}-1 \\
N
\end{array}\right) \prod_{u=2}^{m}\left(\frac{\eta_{u}}{\eta_{u}-\eta_{1}}\right)^{v_{u}} \\
& \times\left[1-\eta_{1} \frac{v_{1}-1}{N+v_{1}-1} K_{1}+\eta_{1}^{2} \frac{\left(v_{1}-1\right)\left(v_{1}-2\right)}{\left(N+v_{1}-1\right)\left(N+v_{1}-2\right)} K_{2}+O\left(\left(\frac{1}{N}\right)^{3}\right)\right] \\
= & \left(\frac{1}{\eta_{1}}\right)^{N}\left(\begin{array}{c}
N+v_{1}-1 \\
N
\end{array}\right) \prod_{u=2}^{m}\left(\frac{\eta_{u}}{\eta_{u}-\eta_{1}}\right)^{v_{u}} \\
& \left.\left.\times\left[1-\eta_{1} \frac{1}{N+v_{1}-1} \tilde{K}_{1}+\eta_{1}^{2} \frac{1}{\left(N+v_{1}-1\right)\left(N+v_{1}-2\right)}+K_{2}\right)^{3}\right)\right] \\
= & \left(\frac{1}{\eta_{1}}\right)^{N}\left(\begin{array}{c}
N+v_{1}-1 \\
N
\end{array}\right) \prod_{u=2}^{m}\left(\frac{\eta_{u}}{\eta_{u}-\eta_{1}}\right)^{v_{u}} a_{N} .
\end{aligned}
$$

The next calculations establish the basis for obtaining asymptotic expansions for the first two cycle time moments.

\section{Proposition 3.2. It holds that}

(i) $\psi^{(M, N+1)}(\theta)$

$$
\begin{aligned}
= & G(M, N)^{-1} \\
& \times \sum_{l=1}^{m} \sum_{a \in Z\left(m, v_{l}-1\right)}(-1)^{a_{l}-v_{l}+1} \eta_{l}^{v_{l}}\left(\frac{1}{\eta_{l}+\theta}\right)^{N+a_{l}+1}\left(\begin{array}{c}
N+a_{l} \\
N
\end{array}\right) \\
& \times\left(\prod_{u=1, u \neq l}^{m} \eta_{u}^{v_{u}}\left(\begin{array}{c}
v_{u}+a_{u}-1 \\
v_{u}-1
\end{array}\right)\left(\frac{1}{\eta_{u}-\eta_{l}}\right)^{v_{u}+a_{u}}\right),
\end{aligned}
$$

(ii) $\mathrm{E}\left[c_{N+1}\right]=\eta_{1}^{-1}\left(N+v_{1}\right) \frac{1}{a_{N}}-\frac{1}{a_{N}} \tilde{K}_{1}+\eta_{1} \frac{1}{a_{N}} \frac{1}{\left(N+v_{1}-1\right)} \tilde{K}_{2}$

$$
+O\left(\left(\frac{1}{N}\right)^{2}\right)
$$

(iii) $\mathrm{E}\left[c_{N+1}\right]=\eta_{1}^{-1}\left(N+v_{1}\right) \frac{1}{a_{N}}-\frac{1}{a_{N}} \tilde{K}_{1}+O\left(\frac{1}{N}\right)$,

(iv) $\mathrm{E}\left[c_{N+1}^{2}\right]=\eta_{1}^{-2}\left(N+v_{1}\right)\left(N+v_{1}+1\right) \frac{1}{a_{N}}-\eta_{1}^{-1}\left(N+v_{1}\right) \frac{1}{a_{N}} \tilde{K}_{1}$

$$
+\frac{1}{a_{N}} \tilde{K}_{2}+O\left(\frac{1}{N}\right) \text {. }
$$


Proof. (i) We have

$$
\begin{aligned}
& \psi^{(M, N+1)}(\theta) G(M, N) \stackrel{(2.2)}{=} G(M, N) \sum_{n \in Z(M, N)} \pi^{(M, N)}(n) \prod_{j=1}^{M}\left(\frac{\mu_{j}}{\mu_{j}+\theta}\right)^{n_{j}+1} \\
& =\left(\prod_{j=1}^{M} \frac{\mu_{j}}{\mu_{j}+\theta}\right) \sum_{n \in Z(M, N)} \prod_{j=1}^{M}\left(\frac{1}{\mu_{j}}\right)^{n_{j}} \prod_{j=1}^{M}\left(\frac{\mu_{j}}{\mu_{j}+\theta}\right)^{n_{j}} \\
& =\prod_{l=1}^{m}\left(\frac{\eta_{l}}{\eta_{l}+\theta}\right)^{v_{l}} \sum_{n \in Z(M, N)} \prod_{j=1}^{M}\left(\frac{1}{\mu_{j}+\theta}\right)^{n_{j}} \\
& \stackrel{(3.7)}{=} \prod_{l=1}^{m}\left(\frac{\eta_{l}}{\eta_{l}+\theta}\right)^{v_{l}} \\
& \times \sum_{l=1}^{m} \sum_{a \in Z\left(m, v_{l}-1\right)}(-1)^{a_{l}-v_{l}+1}\left(\frac{1}{\eta_{l}+\theta}\right)^{N-v_{l}+a_{l}+1}\left(\begin{array}{c}
N+a_{l} \\
N
\end{array}\right) \\
& \times\left(\prod_{u=1, u \neq l}^{m}\left(\eta_{u}+\theta\right)^{v_{u}}\left(\begin{array}{c}
v_{u}+a_{u}-1 \\
v_{u}-1
\end{array}\right)\left(\frac{1}{\eta_{u}-\eta_{l}}\right)^{v_{u}+a_{u}}\right) \\
& =\sum_{l=1}^{m} \sum_{a \in Z\left(m, v_{l}-1\right)}(-1)^{a_{l}-v_{l}+1} \eta_{l}^{v_{l}}\left(\frac{1}{\eta_{l}+\theta}\right)^{N+a_{l}+1}\left(\begin{array}{c}
N+a_{l} \\
N
\end{array}\right) \\
& \times\left(\prod_{u=1, u \neq l}^{m} \eta_{u}^{v_{u}}\left(\begin{array}{c}
v_{u}+a_{u}-1 \\
v_{u}-1
\end{array}\right)\left(\frac{1}{\eta_{u}-\eta_{l}}\right)^{v_{u}+a_{u}}\right) .
\end{aligned}
$$

(ii) Since $\mathrm{E}\left[c_{N+1}\right]=-\partial \psi^{(M, N+1)}(\theta) /\left.\partial \theta\right|_{\theta=0}$, we first calculate $\partial \psi^{(M, N+1)}(\theta) / \partial \theta$ :

$$
\frac{\partial}{\partial \theta} \psi^{(M, N+1)}(\theta)
$$

$$
\begin{aligned}
\stackrel{(3.12)}{=}- & G(M, N)^{-1} \\
\times & \sum_{l=1}^{m} \sum_{a \in Z\left(m, v_{l}-1\right)}(-1)^{a_{l}-v_{l}+1} \eta_{l}^{v_{l}}\left(N+a_{l}+1\right)\left(\frac{1}{\eta_{l}+\theta}\right)^{N+a_{l}+2}\left(\begin{array}{c}
N+a_{l} \\
N
\end{array}\right) \\
& \times\left(\prod_{u=1, u \neq l}^{m} \eta_{u}^{v_{u}}\left(\begin{array}{c}
v_{u}+a_{u}-1 \\
v_{u}-1
\end{array}\right)\left(\frac{1}{\eta_{u}-\eta_{l}}\right)^{v_{u}+a_{u}}\right) .
\end{aligned}
$$

So

$$
\begin{aligned}
& \mathrm{E}\left[c_{N+1}\right]=-\left.\frac{\partial}{\partial \theta} \psi^{(M, N+1)}(\theta)\right|_{\theta=0} \\
& \stackrel{(3.16)}{=} G(M, N)^{-1} \\
& \times \sum_{l=1}^{m} \sum_{a \in Z\left(m, v_{l}-1\right)}(-1)^{a_{l}-v_{l}+1}\left(N+a_{l}+1\right)\left(\frac{1}{\eta_{l}}\right)^{N-v_{l}+a_{l}+2}\left(\begin{array}{c}
N+a_{l} \\
N
\end{array}\right) \\
& \times\left(\prod_{u=1, u \neq l}^{m} \eta_{u}^{v_{u}}\left(\begin{array}{c}
v_{u}+a_{u}-1 \\
v_{u}-1
\end{array}\right)\left(\frac{1}{\eta_{u}-\eta_{l}}\right)^{v_{u}+a_{u}}\right)
\end{aligned}
$$




$$
\begin{aligned}
& =G(M, N)^{-1} \\
& \times \sum_{l=2}^{m} \sum_{a \in Z\left(m, v_{l}-1\right)}(-1)^{a_{l}-v_{l}+1}\left(N+a_{l}+1\right)\left(\frac{1}{\eta_{l}}\right)^{N-v_{l}+a_{l}+2}\left(\begin{array}{c}
N+a_{l} \\
N
\end{array}\right) \\
& \times\left(\prod_{u=1, u \neq l}^{m} \eta_{u}^{v_{u}}\left(\begin{array}{c}
v_{u}+a_{u}-1 \\
v_{u}-1
\end{array}\right)\left(\frac{1}{\eta_{u}-\eta_{l}}\right)^{v_{u}+a_{u}}\right) \\
& +G(M, N)^{-1} \\
& \times \sum_{a \in Z\left(m, v_{1}-1\right), a_{1}<v_{1}-3}(-1)^{a_{1}-v_{1}+1}\left(N+a_{1}+1\right)\left(\frac{1}{\eta_{1}}\right)^{N-v_{1}+a_{1}+2}\left(\begin{array}{c}
N+a_{1} \\
N
\end{array}\right) \\
& \times\left(\prod_{u=2}^{m} \eta_{u}^{v_{u}}\left(\begin{array}{c}
v_{u}+a_{u}-1 \\
v_{u}-1
\end{array}\right)\left(\frac{1}{\eta_{u}-\eta_{1}}\right)^{v_{u}+a_{u}}\right) \\
& +G(M, N)^{-1} \\
& \times \sum_{a \in Z\left(m, \nu_{1}-1\right), a_{1} \geq v_{1}-3}(-1)^{a_{1}-v_{1}+1}\left(N+a_{1}+1\right)\left(\frac{1}{\eta_{1}}\right)^{N-v_{1}+a_{1}+2}\left(\begin{array}{c}
N+a_{1} \\
N
\end{array}\right) \\
& \times\left(\prod_{u=2}^{m} \eta_{u}^{v_{u}}\left(\begin{array}{c}
v_{u}+a_{u}-1 \\
v_{u}-1
\end{array}\right)\left(\frac{1}{\eta_{u}-\eta_{1}}\right)^{v_{u}+a_{u}}\right) .
\end{aligned}
$$

Applying (3.11) leads to the result that the first two sums are of order $O\left((1 / N)^{2}\right)$; therefore,

$$
\begin{aligned}
\mathrm{E}\left[c_{N+1}\right]= & G(M, N)^{-1}\left(N+v_{1}\right)\left(\frac{1}{\eta_{1}}\right)^{N+1}\left(\begin{array}{c}
N+v_{1}-1 \\
N
\end{array}\right) \prod_{u=2}^{m}\left(\frac{\eta_{u}}{\eta_{u}-\eta_{1}}\right)^{v_{u}} \\
& -G(M, N)^{-1}\left(N+v_{1}-1\right)\left(\frac{1}{\eta_{1}}\right)^{N}\left(\begin{array}{c}
N+v_{1}-2 \\
N
\end{array}\right)\left(\prod_{u=2}^{m}\left(\frac{\eta_{u}}{\eta_{u}-\eta_{1}}\right)^{v_{u}}\right) K_{1} \\
& +G(M, N)^{-1}\left(N+v_{1}-2\right)\left(\frac{1}{\eta_{1}}\right)^{N-1}\left(\begin{array}{c}
N+v_{1}-3 \\
N
\end{array}\right)\left(\prod_{u=2}^{m}\left(\frac{\eta_{u}}{\eta_{u}-\eta_{1}}\right)^{v_{u}}\right) K_{2} \\
& +O\left(\left(\frac{1}{N}\right)^{2}\right) \\
\stackrel{(3.11)}{=} & \left(N+v_{1}\right) \eta_{1}^{-1} \frac{1}{a_{N}}-\frac{1}{a_{N}} \frac{\left(N+v_{1}-1\right)\left(v_{1}-1\right)}{N+v_{1}-1} K_{1} \\
& +\eta_{1} \frac{1}{a_{N}} \frac{\left(N+v_{1}-2\right)\left(v_{1}-1\right)\left(v_{1}-2\right)}{\left(N+v_{1}-1\right)\left(N+v_{1}-2\right)} K_{2}+O\left(\left(\frac{1}{N}\right)^{2}\right) \\
= & \left(N+v_{1}\right) \eta_{1}^{-1} \frac{1}{a_{N}}-\frac{1}{a_{N}} \tilde{K}_{1}+\eta_{1} \frac{1}{a_{N}} \frac{1}{\left(N+v_{1}-1\right)} \tilde{K}_{2}+O\left(\left(\frac{1}{N}\right)^{2}\right) .
\end{aligned}
$$

(iii) This is an immediate corollary of (3.13).

(iv) Since

$$
\mathrm{E}\left[c_{N+1}^{2}\right]=\left.\frac{\partial^{2}}{\partial \theta^{2}} \psi^{(M, N+1)}(\theta)\right|_{\theta=0},
$$


we first have to calculate $\partial^{2} \psi^{(M, N+1)}(\theta) / \partial \theta^{2}$ :

$$
\begin{aligned}
& \frac{\partial^{2}}{\partial \theta^{2}} \psi^{(M, N+1)}(\theta) \stackrel{(3.16)}{=} G(M, N)^{-1} \\
& \times \sum_{l=1}^{m} \sum_{a \in Z\left(m, v_{l}-1\right)}(-1)^{a_{l}-v_{l}+1} \eta_{l}^{v_{l}}\left(N+a_{l}+1\right)\left(N+a_{l}+2\right) \\
& \times\left(\frac{1}{\eta_{l}+\theta}\right)^{N+a_{l}+3}\left(\begin{array}{c}
N+a_{l} \\
N
\end{array}\right) \\
& \times\left(\prod_{u=1, u \neq l}^{m} \eta_{u}^{v_{u}}\left(\begin{array}{c}
v_{u}+a_{u}-1 \\
v_{u}-1
\end{array}\right)\left(\frac{1}{\eta_{u}-\eta_{l}}\right)^{v_{u}+a_{u}}\right) .
\end{aligned}
$$

Applying this result yields

$$
\begin{aligned}
\mathrm{E}\left[c_{N+1}^{2}\right]= & \left.\frac{\partial^{2}}{\partial \theta^{2}} \psi^{(M, N+1)}(\theta)\right|_{\theta=0} \\
= & G(M, N)^{-1} \\
& \times \sum_{l=1}^{m} \sum_{a \in Z\left(m, v_{l}-1\right)}(-1)^{a_{l}-v_{l}+1}\left(N+a_{l}+1\right)\left(N+a_{l}+2\right) \\
\times & \left(\frac{1}{\eta_{l}}\right)^{N-v_{l}+a_{l}+3}\left(\begin{array}{c}
N+a_{l} \\
N
\end{array}\right) \\
\times & \left(\prod_{u=1, u \neq l}^{m} \eta_{u}^{v_{u}}\left(\begin{array}{c}
v_{u}+a_{u}-1 \\
v_{u}-1
\end{array}\right)\left(\frac{1}{\eta_{u}-\eta_{l}}\right)\right. \\
= & \left(N+v_{1}\right)\left(N+v_{1}+1\right) \eta_{1}^{-2} \frac{1}{a_{N}}-\left(N+v_{1}-1\right)\left(N+v_{1}\right) \eta_{1}^{-1} \frac{1}{a_{N}} \frac{1}{N+v_{1}-1} \\
& +\left(N+v_{1}-2\right)\left(N+v_{1}-1\right) \frac{1}{a_{N}} \frac{1}{\left(N+v_{1}-1\right)\left(N+v_{1}-2\right)} \tilde{K}_{2}+O\left(\frac{1}{N}\right) \\
= & \left(N+v_{1}\right)\left(N+v_{1}+1\right) \eta_{1}^{-2} \frac{1}{a_{N}}-\left(N+v_{1}\right) \eta_{1}^{-1} \frac{1}{a_{N}} \tilde{K}_{1}+\frac{1}{a_{N}} \tilde{K}_{2}+O\left(\frac{1}{N}\right) .
\end{aligned}
$$

We are now in a position to prove Theorems 3.1 and 3.2.

Proof of Theorem 3.1. Note that $\mu_{1}=\eta_{1}$. Then

$$
\begin{aligned}
& \lim _{N \rightarrow \infty}\left(\mathrm{E}\left[c_{N+1}\right]-\eta_{1}^{-1}\left(N+v_{1}\right)\right) \\
& \stackrel{(\text { 3.14) }}{=} \lim _{N \rightarrow \infty}\left(\eta_{1}^{-1}\left(N+v_{1}\right) \frac{1}{a_{N}}-\frac{1}{a_{N}} \tilde{K}_{1}+O\left(\frac{1}{N}\right)-\eta_{1}^{-1}\left(N+v_{1}\right)\right) \\
& \quad=\eta_{1}^{-1} \lim _{N \rightarrow \infty}\left(\frac{N\left(1-a_{N}\right)}{a_{N}}\right)+\lim _{N \rightarrow \infty}\left(\frac{v_{1}\left(1-a_{N}\right)}{a_{N}}\right)-\lim _{N \rightarrow \infty} \frac{\tilde{K}_{1}}{a_{N}} \\
& \quad \stackrel{(3.9)}{=} 0,
\end{aligned}
$$

and the theorem follows. 
Proof of Theorem 3.2. Since $\mu_{1}=\eta_{1}$, it is sufficient to prove that $\lim _{N \rightarrow \infty}\left(\operatorname{var}\left(c_{N+1}\right)-\right.$ $\left.\eta_{1}^{-2}\left(N+v_{1}\right)\right)=0$. We have

$$
\begin{aligned}
\mathrm{E}^{2}\left[c_{N+1}\right] \stackrel{(3.13)}{=} & \left(\eta_{1}^{-1}\left(N+v_{1}\right) \frac{1}{a_{N}}-\frac{1}{a_{N}} \tilde{K}_{1}+\eta_{1} \frac{1}{a_{N}} \frac{1}{\left(N+v_{1}-1\right)} \tilde{K}_{2}+O\left(\left(\frac{1}{N}\right)^{2}\right)\right)^{2} \\
= & \left(\eta_{1}^{-1}\left(N+v_{1}\right) \frac{1}{a_{N}}-\frac{1}{a_{N}} \tilde{K}_{1}+\eta_{1} \frac{1}{a_{N}} \frac{1}{\left(N+v_{1}-1\right)} \tilde{K}_{2}\right)^{2}+O\left(\frac{1}{N}\right) \\
= & \left(\eta_{1}^{-1}\left(N+v_{1}\right) \frac{1}{a_{N}}-\frac{1}{a_{N}} \tilde{K}_{1}\right)^{2} \\
& +2\left(\eta_{1}^{-1}\left(N+v_{1}\right) \frac{1}{a_{N}}-\frac{1}{a_{N}} \tilde{K}_{1}\right)\left(\eta_{1} \frac{1}{a_{N}} \frac{1}{\left(N+v_{1}-1\right)} \tilde{K}_{2}\right) \\
& +\left(\eta_{1} \frac{1}{a_{N}} \frac{1}{\left(N+v_{1}-1\right)} \tilde{K}_{2}\right)^{2}+O\left(\frac{1}{N}\right) \\
= & \left(\eta_{1}^{-1}\left(N+v_{1}\right) \frac{1}{a_{N}}-\frac{1}{a_{N}} \tilde{K}_{1}\right)^{2}+2 \frac{1}{a_{N}^{2}} \frac{N+v_{1}}{N+v_{1}-1} \tilde{K}_{2}+O\left(\frac{1}{N}\right) \\
= & \eta_{1}^{-2}\left(N+v_{1}\right)^{2} \frac{1}{a_{N}^{2}}-2 \eta_{1}^{-1}\left(N+v_{1}\right) \frac{1}{a_{N}^{2}} \tilde{K}_{1}+\frac{1}{a_{N}^{2}} \tilde{K}_{1}^{2} \\
& +2 \frac{1}{a_{N}^{2}} \frac{N+v_{1}}{N+v_{1}-1} \tilde{K}_{2}+O\left(\frac{1}{N}\right)
\end{aligned}
$$

and

$$
\begin{aligned}
& \lim _{N \rightarrow \infty}\left(\operatorname{var}\left(c_{N+1}\right)-\eta_{1}^{-2}\left(N+v_{1}\right)\right) \\
& =\lim _{N \rightarrow \infty}\left(\mathrm{E}\left[c_{N+1}^{2}\right]-\mathrm{E}^{2}\left[c_{N+1}\right]-\eta_{1}^{-2}\left(N+v_{1}\right)\right) \\
& \stackrel{(3.15)}{=} \lim _{N \rightarrow \infty}\left[\eta_{1}^{-2}\left(N+v_{1}\right)\left(N+v_{1}+1\right) \frac{1}{a_{N}}-\eta_{1}^{-1}\left(N+v_{1}\right) \frac{1}{a_{N}} \tilde{K}_{1}+\frac{1}{a_{N}} \tilde{K}_{2}\right. \\
& \quad-\eta_{1}^{-2}\left(N+v_{1}\right)^{2} \frac{1}{a_{N}^{2}}+2 \eta_{1}^{-1}\left(N+v_{1}\right) \frac{1}{a_{N}^{2}} \tilde{K}_{1}-\frac{1}{a_{N}^{2}} \tilde{K}_{1}^{2} \\
& \left.\quad-2 \frac{1}{a_{N}^{2}} \frac{N+v_{1}}{N+v_{1}-1} \tilde{K}_{2}-\eta_{1}^{-2}\left(N+v_{1}\right)\right] \\
& \quad \lim _{N \rightarrow \infty}\left[\eta_{1}^{-2}\left(N+v_{1}\right)^{2} \frac{1}{a_{N}}-\eta_{1}^{-2}\left(N+v_{1}\right)^{2} \frac{1}{a_{N}^{2}}-\eta_{1}^{-1}\left(N+v_{1}\right) \frac{1}{a_{N}} \tilde{K}_{1}\right. \\
& \left.\quad+2 \eta_{1}^{-1}\left(N+v_{1}\right) \frac{1}{a_{N}^{2}} \tilde{K}_{1}\right] \\
& +\lim _{N \rightarrow \infty}\left(\eta_{1}^{-2}\left(N+v_{1}\right) \frac{1}{a_{N}}-\eta_{1}^{-2}\left(N+v_{1}\right)\right)-\tilde{K}_{1}^{2}-\tilde{K}_{2} .
\end{aligned}
$$

Note that

$$
\lim _{N \rightarrow \infty}\left(\eta_{1}^{-2}\left(N+v_{1}\right) \frac{1}{a_{N}}-\eta_{1}^{-2}\left(N+v_{1}\right)\right)=\eta_{1}^{-2} \lim _{N \rightarrow \infty}\left(\frac{N\left(1-a_{N}\right)}{a_{N}}\right) \stackrel{(3.9)}{=} \eta_{1}^{-1} \tilde{K}_{1} .
$$


Also, note that, using Lemma 3.2 at $\stackrel{(* *),}{=}$,

$$
\begin{aligned}
\eta_{1}^{-2}(N & \left.+v_{1}\right)^{2} \frac{1}{a_{N}}-\eta_{1}^{-2}\left(N+v_{1}\right)^{2} \frac{1}{a_{N}^{2}} \\
= & \eta_{1}^{-2} \frac{1}{a_{N}^{2}}\left(N+v_{1}\right)^{2}\left(a_{N}-1\right) \\
\stackrel{(* *)}{=} & \eta_{1}^{-2} \frac{1}{a_{N}^{2}}\left(N^{2}+2 N v_{1}+v_{1}^{2}\right) \\
& \times\left(-\eta_{1} \frac{1}{N+v_{1}-1} \tilde{K}_{1}+\eta_{1}^{2} \frac{1}{\left(N+v_{1}-1\right)\left(N+v_{1}-2\right)} \tilde{K}_{2}+O\left(\left(\frac{1}{N}\right)^{3}\right)\right) \\
= & -\eta_{1}^{-1} \frac{1}{a_{N}^{2}} \frac{N^{2}}{N+v_{1}-1} \tilde{K}_{1}+\frac{1}{a_{N}^{2}} \frac{N^{2}}{\left(N+v_{1}-1\right)\left(N+v_{1}-2\right)} \tilde{K}_{2} \\
& \quad-2 \eta_{1}^{-1} v_{1} \frac{N}{N+v_{1}-1} \tilde{K}_{1}+O\left(\frac{1}{N}\right) .
\end{aligned}
$$

Inserting (3.18) and (3.19) into (3.17) leads to

$$
\begin{aligned}
\lim _{N \rightarrow \infty}( & \left.\operatorname{var}\left(c_{N+1}\right)-\eta_{1}^{-2}\left(N+v_{1}\right)\right) \\
= & \lim _{N \rightarrow \infty}\left[-\eta_{1}^{-1} \frac{1}{a_{N}^{2}} \frac{N^{2}}{N+v_{1}-1} \tilde{K}_{1}-\eta_{1}^{-1}\left(N+v_{1}\right) \frac{1}{a_{N}} \tilde{K}_{1}+2 \eta_{1}^{-1}\left(N+v_{1}\right) \frac{1}{a_{N}^{2}} \tilde{K}_{1}\right] \\
& +\eta_{1}^{-1} \tilde{K}_{1}-2 \eta_{1}^{-1} v_{1} \tilde{K}_{1}-\tilde{K}_{1}^{2}+\tilde{K}_{2}-\tilde{K}_{2} \\
= & \eta_{1}^{-1} \tilde{K}_{1} \lim _{N \rightarrow \infty}[\frac{1}{a_{N}^{2}} \underbrace{\frac{\left(N+v_{1}\right)\left(N+v_{1}-1\right)-N^{2}}{N+v_{1}-1}}_{\rightarrow 2 v_{1}-1}+\underbrace{\left.\frac{1}{a_{N}^{2}}\left(N+v_{1}\right)\left(1-a_{N}\right)\right]}_{\rightarrow \eta_{1} \tilde{K}_{1}} \\
& +\eta_{1}^{-1} \tilde{K}_{1}-2 \eta_{1}^{-1} v_{1} \tilde{K}_{1}-\tilde{K}_{1}^{2} \\
= & 0 .
\end{aligned}
$$

\subsection{Central limit theorem for cycle times}

The following theorem removes the requirement of distinct service rates at all nodes in Theorem 4.1 of [10]. It turns out that the methods needed for the proof are completely different.

Theorem 3.3. (Central limit theorem for the cycle time.) Let $\mu_{1} \leq \cdots \leq \mu_{M}$. Then

$$
\frac{c_{N}-\mathrm{E}\left[c_{N}\right]}{\sqrt{\operatorname{var}\left(c_{N}\right)}} \stackrel{\mathrm{D}}{\rightarrow} X \sim \mathcal{N}(0,1) \quad \text { as } N \rightarrow \infty .
$$

Proof. We shall utilize from Slutsky's theorem [1, p. 461] the following facts. Let $X, X_{n}$, and $Y_{n}, n \geq 1$, be real-valued random variables so that $X_{n} \stackrel{\mathrm{D}}{\rightarrow} X$ and $Y_{n} \stackrel{\mathrm{P}}{\rightarrow} c$ for some $c \in \mathbb{R}$. Then it holds that

$$
X_{n}+Y_{n} \stackrel{\mathrm{D}}{\rightarrow} X+c, \quad X_{n} Y_{n} \stackrel{\mathrm{D}}{\rightarrow} X c .
$$

Recall from (3.1) that $c_{N}$ can be expressed as $c_{N}=\delta_{N}+\rho_{N}$, with $\delta_{N}$ the sum of $N$ i.i.d. $\exp \left(\mu_{1}\right)$-distributed service times and $\rho_{N}$ the cumulative idle time. Therefore, the normalized 
cycle time can be written as

$$
\frac{c_{N}-\mathrm{E}\left[c_{N}\right]}{\sqrt{\operatorname{var}\left(c_{N}\right)}}=\frac{\delta_{N}-\mathrm{E}\left[\delta_{N}\right]+\rho_{N}-\mathrm{E}\left[\rho_{N}\right]}{\sqrt{\operatorname{var}\left(c_{N}\right)}}=\frac{\delta_{N}-\mathrm{E}\left[\delta_{N}\right]}{\sqrt{\operatorname{var}\left(\delta_{N}\right)}} \frac{\sqrt{\operatorname{var}\left(\delta_{N}\right)}}{\sqrt{\operatorname{var}\left(c_{N}\right)}}+\frac{\rho_{N}-\mathrm{E}\left[\rho_{N}\right]}{\sqrt{\operatorname{var}\left(c_{N}\right)}} .
$$

First, note that, because of the central limit theorem for i.i.d. random variables,

$$
\frac{\delta_{N}-\mathrm{E}\left[\delta_{N}\right]}{\sqrt{\operatorname{var}\left(\delta_{N}\right)}} \stackrel{\mathrm{D}}{\rightarrow} X \sim \mathcal{N}(0,1) \quad \text { as } N \rightarrow \infty
$$

Since $\operatorname{var}\left(\delta_{N}\right)=\mu_{1}^{-2} N \rightarrow \infty$ as $N \rightarrow \infty$ and $\lim _{N \rightarrow \infty}\left|\operatorname{var}\left(c_{N}\right)-\operatorname{var}\left(\delta_{N}\right)\right|=$ constant (see (3.4)), it holds that

$$
\lim _{N \rightarrow \infty} \frac{\sqrt{\operatorname{var}\left(\delta_{N}\right)}}{\sqrt{\operatorname{var}\left(c_{N}\right)}}=1 .
$$

Combining (3.22) and (3.23) and applying (3.21), we obtain

$$
\frac{\delta_{N}-\mathrm{E}\left[\delta_{N}\right]}{\sqrt{\operatorname{var}\left(\delta_{N}\right)}} \frac{\sqrt{\operatorname{var}\left(\delta_{N}\right)}}{\sqrt{\operatorname{var}\left(c_{N}\right)}} \stackrel{\mathrm{D}}{\rightarrow} X \sim \mathcal{N}(0,1) \quad \text { as } N \rightarrow \infty .
$$

Finally, let us analyze the last term, $\left(\rho_{N}-\mathrm{E}\left[\rho_{N}\right]\right) / \sqrt{\operatorname{var}\left(c_{N}\right)}$. It holds that

$$
\mathrm{E}\left[\left|\frac{\rho_{N}-\mathrm{E}\left[\rho_{N}\right]}{\sqrt{\operatorname{var}\left(c_{N}\right)}}\right|\right]=\frac{\mathrm{E}\left[\left|\rho_{N}-\mathrm{E}\left[\rho_{N}\right]\right|\right]}{\sqrt{\operatorname{var}\left(c_{N}\right)}} \leq \frac{2 \mathrm{E}\left[\rho_{N}\right]}{\sqrt{\operatorname{var}\left(c_{N}\right)}} \rightarrow 0 \quad \text { as } N \rightarrow \infty
$$

since $\operatorname{var}\left(c_{N}\right) \rightarrow \infty$ as $N \rightarrow \infty$ and

$$
\lim _{N \rightarrow \infty} \mathrm{E}\left[\rho_{N}\right]=\lim _{N \rightarrow \infty}\left(\mathrm{E}\left[c_{N}\right]-\mathrm{E}\left[\delta_{N}\right]\right)=\lim _{N \rightarrow \infty}\left(\mathrm{E}\left[c_{N}\right]-N \mu_{1}^{-1}\right) \stackrel{(3.3)}{=} \text { constant. }
$$

Applying the Markov inequality,

$$
\mathrm{P}\left(\left|\frac{\rho_{N}-\mathrm{E}\left[\rho_{N}\right]}{\sqrt{\operatorname{var}\left(c_{N}\right)}}\right| \geq \varepsilon\right) \leq \frac{\mathrm{E}\left[\left|\left(\rho_{N}-\mathrm{E}\left[\rho_{N}\right]\right) / \sqrt{\operatorname{var}\left(c_{N}\right)}\right|\right]}{\varepsilon} \rightarrow 0 \quad \text { as } N \rightarrow \infty \text { for all } \varepsilon>0,
$$

it follows that

$$
\frac{\rho_{N}-\mathrm{E}\left[\rho_{N}\right]}{\sqrt{\operatorname{var}\left(c_{N}\right)}} \stackrel{\mathrm{P}}{\rightarrow} 0 \quad \text { as } N \rightarrow \infty .
$$

Combining (3.24) and (3.25) and applying (3.21) yields (3.20).

\section{Single bottleneck case versus multiple bottleneck case}

We now analyze how the filling behaviour of the networks depends on the number of bottleneck nodes. We noted in Corollary 3.1 the discontinuity of the asymptotic idle time behaviour when varying the service rates, i.e. $\lim _{N \rightarrow \infty} \mathrm{E}\left[\rho_{N}\right]=\left(v_{1}-1\right) \mu_{1}^{-1}$, which is greater than 0 for $\nu_{1}>1$ and 0 for $\nu_{1}=1$. For pairwise distinct service rates $\mu_{1}<\cdots<\mu_{M}$, it even holds that $\mathrm{E}\left[\rho_{N}\right]=N \mu_{1}^{-1} O\left(\left(\mu_{1} / \mu_{2}\right)^{N}\right)$; cf. [3].

For the cumulative idle time $\rho_{N}$, it makes a major difference whether the network has a single bottleneck node or multiple bottleneck nodes. In the case of a single bottleneck, the cumulative idle time of a bottleneck node during a customer's cycle does not only converge stochastically to 0 for $N \rightarrow \infty$, but the rate of convergence is very high. Thus, for large values of $N$, the bottleneck node will almost never be empty. Also, since $c_{N}=\delta_{N}+\rho_{N}$ and $\rho_{N}$ is converging 
stochastically to 0 , for large values of $N$, the cycle time $c_{N}$ can be approximated by $\delta_{N}$, the sum of $N$ i.i.d. $\exp \left(\mu_{1}\right)$-distributed variables. In contrast, in the case of multiple bottleneck nodes, the expected cumulative idle time converges towards a positive constant, i.e. during one cycle, the bottleneck node $Q[1]$ will on average be empty for a positive time period. By symmetry, this also holds for the other bottlenecks. For the filling behaviour of bottleneck nodes, we observe a similar dichotomy.

Theorem 4.1. Let $Q[i]$ be a bottleneck node. Then it holds that

$$
\mathrm{P}\left(X_{i}^{(M, N)} \geq r\right)= \begin{cases}1-O\left(\frac{1}{N}\right) & \text { for } v_{1}>1, \\ 1-N^{\nu_{2}-1} O\left(\left(\frac{\mu_{1}}{\mu_{2}}\right)^{N}\right) & \text { for } v_{1}=1,\end{cases}
$$

and the equation is sharp with respect to the speed of convergence.

Proof. Without loss of generality, we assume that $Q[i]=Q[1]$. We then have

$$
\begin{aligned}
\mathrm{P}\left(X_{1}^{(M, N)}<r\right) & =\sum_{n \in Z(M, N)} \pi(n) \mathrm{P}\left(X_{1}^{(M, N)}<r \mid X^{(M, N)}=n\right) \\
& \stackrel{(2.1)}{=} \sum_{n_{1}=0}^{r-1}\left[\sum_{n_{2}+\cdots+n_{M}=N-n_{1}} G(M, N)^{-1} \prod_{j=1}^{M}\left(\frac{1}{\mu_{j}}\right)^{n_{j}}\right] \\
& =\sum_{n_{1}=0}^{r-1}\left(\frac{1}{\mu_{1}}\right)^{n_{1}}\left[\frac{\sum_{n_{2}+\cdots+n_{M}=N-n_{1}} \prod_{j=2}^{M}\left(1 / \mu_{j}\right)^{n_{j}}}{G(M, N)}\right] .
\end{aligned}
$$

Let us first consider the case of multiple bottlenecks, i.e. $v_{1}>1$. Note that

$$
\sum_{n_{2}+\cdots+n_{M}=N-n_{1}} \prod_{j=2}^{M}\left(\frac{1}{\mu_{j}}\right)^{n_{j}}
$$

is the normalizing constant of a network with the same service rates, but with one bottleneck node less and $n_{1}$ customers less. We therefore define, for $j=1, \ldots, m$,

$$
\tilde{v_{j}}= \begin{cases}v_{1}-1 & \text { for } j=1 \\ v_{j} & \text { for } j \neq 1\end{cases}
$$

It follows that

$$
\begin{aligned}
& \sum_{n_{2}+\cdots+n_{M}=N-n_{1}} \prod_{j=2}^{M}\left(\frac{1}{\mu_{j}}\right)^{n_{j}} \\
& \stackrel{(3.7)}{=} \sum_{l=1}^{m} \sum_{a \in Z\left(m, \tilde{v_{l}}-1\right)}(-1)^{a_{l}-\tilde{v_{l}}+1}\left(\frac{1}{\eta_{l}}\right)^{N-n_{1}-\tilde{v}_{l}+a_{l}+1}\left(\begin{array}{c}
N-n_{1}+a_{l} \\
N-n_{1}
\end{array}\right) \\
& \times\left(\prod_{u=1, u \neq l}^{m} \eta_{u}^{\tilde{v_{u}}}\left(\begin{array}{c}
\tilde{v_{u}}+a_{u}-1 \\
\tilde{v_{u}}-1
\end{array}\right)\left(\frac{1}{\eta_{u}-\eta_{l}}\right)^{\tilde{v_{u}}+a_{u}}\right) .
\end{aligned}
$$


From (3.11), we know that

$$
G(M, N)=\left(\frac{1}{\eta_{1}}\right)^{N}\left(\begin{array}{c}
N+v_{1}-1 \\
N
\end{array}\right)\left(\prod_{u=2}^{m}\left(\frac{\eta_{u}}{\eta_{u}-\eta_{1}}\right)^{v_{u}}\right) a_{N},
$$

with $a_{N}=1+O(1 / N)$. Note that, for $2 \leq l \leq m$ and $a \in Z\left(m, \tilde{v}_{l}-1\right)$, it holds that

$$
\begin{aligned}
& G(M, N)^{-1}(-1)^{a_{l}-\tilde{v}_{l}+1}\left(\frac{1}{\eta_{l}}\right)^{N-n_{1}-\tilde{v}_{l}+a_{l}+1}\left(\begin{array}{c}
N-n_{1}+a_{l} \\
N-n_{1}
\end{array}\right) \\
& \times\left(\prod_{u=1, u \neq l}^{m} \eta_{u}^{\tilde{v_{u}}}\left(\begin{array}{c}
\tilde{v_{u}}+a_{u}-1 \\
\tilde{v_{u}}-1
\end{array}\right)\left(\frac{1}{\eta_{u}-\eta_{l}}\right)^{\tilde{v_{u}}+a_{u}}\right) \\
& =\left[(-1)^{a_{l}-\tilde{\nu}_{l}+1}\left(\frac{1}{\eta_{l}}\right)^{N-n_{1}-\tilde{\nu}_{l}+a_{l}+1}\left(\begin{array}{c}
N-n_{1}+a_{l} \\
N-n_{1}
\end{array}\right)\right. \\
& \left.\times\left(\prod_{u=1, u \neq l}^{m} \eta_{u}^{\tilde{v_{u}}}\left(\begin{array}{c}
\tilde{v_{u}}+a_{u}-1 \\
\tilde{v_{u}}-1
\end{array}\right)\left(\frac{1}{\eta_{u}-\eta_{l}}\right)^{\tilde{v_{u}}+a_{u}}\right)\right] \\
& \times\left[\left(\frac{1}{\eta_{1}}\right)^{N}\left(\begin{array}{c}
N+v_{1}-1 \\
N
\end{array}\right)\left(\prod_{u=2}^{m}\left(\frac{\eta_{u}}{\eta_{u}-\eta_{1}}\right)^{v_{u}}\right) a_{N}\right]^{-1} \\
& =\text { constant }\left(\frac{\eta_{1}}{\eta_{l}}\right)^{N} \frac{\left(\begin{array}{c}
N-n_{1}+a_{l} \\
N-n_{1}
\end{array}\right)}{\left(\begin{array}{c}
N+v_{1}-1 \\
N
\end{array}\right)} \frac{1}{a_{N}} \\
& =O\left(\left(\frac{1}{N}\right)^{2}\right)
\end{aligned}
$$

since

$$
\lim _{N \rightarrow \infty} N^{p}\left(\frac{\eta_{1}}{\eta_{l}}\right)^{N}=0, \quad p \in \mathbb{N}
$$

Because of (4.3), which holds for all $p \in \mathbb{N}, 2 \leq l \leq m$, it follows that

$$
\begin{aligned}
& G(M, N)^{-1} \sum_{n_{2}+\cdots+n_{M}=N-n_{1}} \prod_{j=2}^{M}\left(\frac{1}{\mu_{j}}\right)^{n_{j}} \\
&=G(M, N)^{-1} \sum_{a \in Z\left(m, \tilde{v_{1}}-1\right)}(-1)^{a_{1}-\tilde{v_{1}}+1}\left(\frac{1}{\eta_{1}}\right)^{N-n_{1}-\tilde{v_{1}+a_{1}+1}}\left(\begin{array}{c}
N-n_{1}+a_{1} \\
N-n_{1}
\end{array}\right) \\
& \quad \times\left(\prod_{u=2}^{m} \eta_{u}^{\tilde{v_{u}}}\left(\begin{array}{c}
\tilde{v_{u}}+a_{u}-1 \\
\tilde{v_{u}}-1
\end{array}\right)\left(\frac{1}{\eta_{u}-\eta_{1}}\right)^{\tilde{v_{u}}+a_{u}}\right)+O\left(\left(\frac{1}{N}\right)^{2}\right) .
\end{aligned}
$$

Only the term with $a_{1}=\tilde{v_{1}}-1=v_{1}-2$ needs to be considered, the rest is of order $O\left((1 / N)^{2}\right)$. 
Therefore,

$$
\begin{aligned}
& G(M, N)^{-1} \sum_{n_{2}+\cdots+n_{M}=N-n_{1}} \prod_{j=2}^{M}\left(\frac{1}{\mu_{j}}\right)^{n_{j}} \\
& =\frac{\left(1 / \eta_{1}\right)^{N-n_{1}}\left(\begin{array}{c}
N-n_{1}+v_{1}-2 \\
N-n_{1}
\end{array}\right) \prod_{u=2}^{m}\left(\eta_{u} /\left(\eta_{u}-\eta_{1}\right)\right)^{v_{u}}}{\left(1 / \eta_{1}\right)^{N}\left(\begin{array}{c}
N+v_{1}-1 \\
N
\end{array}\right)\left(\prod_{u=2}^{m}\left(\eta_{u} /\left(\eta_{u}-\eta_{1}\right)\right)^{v_{u}}\right)(1+O(1 / N))}+O\left(\left(\frac{1}{N}\right)^{2}\right) \\
& =\frac{\left(1 / \eta_{1}\right)^{-n_{1}}\left(\begin{array}{c}
N-n_{1}+v_{1}-2 \\
N-n_{1}
\end{array}\right)}{\left(\begin{array}{c}
N+v_{1}-1 \\
N
\end{array}\right)(1+O(1 / N))}+O\left(\left(\frac{1}{N}\right)^{2}\right) \\
& =\frac{O(1 / N)}{1+O(1 / N)}+O\left(\left(\frac{1}{N}\right)^{2}\right) \\
& =O\left(\frac{1}{N}\right) \text {. }
\end{aligned}
$$

Substituting (4.5) into (4.2) leads to the first part of (4.1). From (4.4), we see that

$$
\lim _{N \rightarrow \infty}\left(N G(M, N)^{-1} \sum_{n_{2}+\cdots+n_{M}=N-n_{1}} \prod_{j=2}^{M}\left(\frac{1}{\mu_{j}}\right)^{n_{j}}\right) \begin{cases}>0 & \text { for } n_{1}<N \\ \geq 0 & \text { for } n_{1}=N .\end{cases}
$$

This shows that, for $v_{1}>1$, it holds that

$$
\mathrm{P}\left(X_{i}^{(M, N)} \geq r\right)=1-O\left(\frac{1}{N}\right) \quad \text { and } \quad \mathrm{P}\left(X_{i}^{(M, N)} \geq r\right) \neq 1-o\left(\frac{1}{N}\right) .
$$

For the case of a single bottleneck, i.e. $v_{1}=1$, we have

$$
\begin{gathered}
\sum_{n_{2}+\cdots+n_{M}=N-n_{1}} \prod_{j=2}^{M}\left(\frac{1}{\mu_{j}}\right)^{n_{j}} \\
\stackrel{(3.7)}{=} \sum_{l=2}^{m} \sum_{a \in Z\left(m, v_{l}-1\right)}(-1)^{a_{l}-v_{l}+1}\left(\frac{1}{\eta_{l}}\right)^{N-n_{1}-v_{l}+a_{l}+1}\left(\begin{array}{c}
N-n_{1}+a_{l} \\
N-n_{1}
\end{array}\right) \\
\times\left(\prod_{u=1, u \neq l}^{m} \eta_{u}^{v_{u}}\left(\begin{array}{c}
v_{u}+a_{u}-1 \\
v_{u}-1
\end{array}\right)\left(\frac{1}{\eta_{u}-\eta_{l}}\right)^{v_{u}+a_{u}}\right) \\
=\sum_{l=2}^{m}\left(\frac{1}{\eta_{l}}\right)^{N} \sum_{a \in Z\left(m, v_{l}-1\right)}(-1)^{a_{l}-v_{l}+1}\left(\frac{1}{\eta_{l}}\right)^{-n_{1}-v_{l}+a_{l}+1}\left(\begin{array}{c}
N-n_{1}+a_{l} \\
N-n_{1}
\end{array}\right) \\
\times\left(\prod_{u=1, u \neq l}^{m} \eta_{u}^{v_{u}}\left(\begin{array}{c}
v_{u}+a_{u}-1 \\
v_{u}-1
\end{array}\right)\left(\frac{1}{\eta_{u}-\eta_{l}}\right)^{v_{u}+a_{u}}\right.
\end{gathered}
$$

It follows, with

$$
G(M, N)=\left(\frac{1}{\eta_{1}}\right)^{N}\left(\prod_{u=2}^{m}\left(\frac{\eta_{u}}{\eta_{u}-\eta_{1}}\right)^{v_{u}}\right) a_{N}
$$


(cf. (3.11)), that

$$
\begin{aligned}
& G(M, N)^{-1} \sum_{n_{2}+\cdots+n_{M}=N-n_{1}} \prod_{j=2}^{M}\left(\frac{1}{\mu_{j}}\right)^{n_{j}} \\
&= \frac{1}{a_{N}} \sum_{l=2}^{m}\left(\frac{\eta_{1}}{\eta_{l}}\right)^{N} \\
& \times \sum_{a \in Z\left(m, v_{l}-1\right)}(-1)^{a_{l}-v_{l}+1}\left(\frac{1}{\eta_{l}}\right)^{-n_{1}-v_{l}+a_{l}+1}\left(\begin{array}{c}
N-n_{1}+a_{l} \\
N-n_{1}
\end{array}\right)\left(\prod_{u=2}^{m}\left(\frac{\eta_{u}}{\eta_{u}-\eta_{1}}\right)^{v_{u}}\right)^{-1} \\
&=\left.\frac{1}{a_{N}}\left(\frac{\eta_{1}}{\eta_{2}}\right)^{N} \sum_{l=2}^{m}\left(\frac{\eta_{2}}{\eta_{l}}\right)^{N} \prod_{u=1, u \neq l}^{v_{u}+a_{u}}\right) \\
& \times \sum_{a \in Z\left(m, v_{l}-1\right)} \eta_{u}^{v_{u}}\left(\begin{array}{c}
v_{u}+a_{u}-1 \\
v_{u}-1
\end{array}\right)\left(\frac{1}{\eta_{u}-\eta_{l}}\right)^{a_{l}-v_{l}+1}\left(\frac{1}{\eta_{l}}\right)^{-n_{1}-v_{l}+a_{l}+1}\left(N-n_{1}+a_{l}\right. \\
& N
\end{aligned}
$$

Since, for all $p \in \mathbb{N}$,

$$
\lim _{N \rightarrow \infty} N^{p}\left(\frac{\eta_{2}}{\eta_{l}}\right)^{N}=0, \quad 3 \leq l \leq m,
$$

only the sum for $l=2$ is of importance and only the summand with $a_{2}=v_{2}-1$ needs to be considered. This leads to

$$
\begin{aligned}
G(M, N)^{-1} \sum_{n_{2}+\cdots+n_{M}=N-n_{1}} \prod_{j=2}^{M}\left(\frac{1}{\mu_{j}}\right)^{n_{j}} & =\frac{1}{a_{N}}\left(\begin{array}{c}
N-n_{1}+v_{2}-1 \\
N-n_{1}
\end{array}\right)\left(\frac{\eta_{1}}{\eta_{2}}\right)^{N}\left[1+O\left(\frac{1}{N}\right)\right] \\
& =N^{\nu_{2}-1} O\left(\left(\frac{\eta_{1}}{\eta_{2}}\right)^{N}\right) \\
& \stackrel{\mu_{2}=\eta_{2}}{=} N^{\nu_{2}-1} O\left(\left(\frac{\mu_{1}}{\mu_{2}}\right)^{N}\right) .
\end{aligned}
$$

Substituting (4.7) into (4.2) leads to the second part of (4.1). We can show that (4.6) implies that

$$
\begin{gathered}
\lim _{N \rightarrow \infty}\left(\left[N^{\nu_{2}-1}\left(\frac{\eta_{1}}{\eta_{2}}\right)^{N}\right]^{-1} G(M, N)^{-1} \sum_{n_{2}+\cdots+n_{M}=N-n_{1}} \prod_{j=2}^{M}\left(\frac{1}{\mu_{j}}\right)^{n_{j}}\right) \\
\begin{cases}>0 & \text { for } n_{1}<N, \\
\geq 0 & \text { for } n_{1}=N .\end{cases}
\end{gathered}
$$

This shows that the second equation of (4.1) is sharp according to the speed. 
From (4.1), $\lim _{N \rightarrow \infty} \mathrm{P}\left(X_{1}^{(M, N)} \geq r\right)=1$ for every $r \in \mathbb{N}$, i.e. bottlenecks are filled up asymptotically. But the way they get filled up is essentially different. In the case of a single bottleneck, we have a very high rate of convergence; in the case of multiple bottlenecks, the rate of convergence is quite low, $O(1 / N)$. The fact that in the multiple bottleneck case, the bottleneck nodes are filled up slower is comprehensible since the customers spread over the bottleneck nodes. However, the size of the difference is surprising.

It is well known [3] that in the single bottleneck case the rate of convergence of the filling behaviour is mainly determined by the quotient $\left(\mu_{1} / \mu_{2}\right)^{N}$. However, the speed of convergence is also influenced by the number of second slowest servers $\left(N^{\nu_{2}-1}\right)$, i.e. the more second slowest servers there are in the network, the more customers will be present at nonbottleneck nodes. This is an interesting result, an interpretation of which can be given as follows. The second slowest servers more strongly resist the fast passing of customers than all other nonbottleneck nodes. Calling these nodes 'semibottlenecks' would make this phenomenon intuitive. The semibottlenecks are the most important hills which customers have to climb up on their way to the single bottleneck. The more hills, the longer the time to reach the bottleneck for almost all customers.

\section{Weak convergence of sojourn times at nonbottleneck nodes}

In the case of a single bottleneck the joint sojourn time distribution for the TC at the nonbottleneck nodes $Q[2], \ldots, Q[J]$ during her cycle converges for $N \rightarrow \infty$ to the joint distribution of the sojourn times of a customer passing this tandem fed by a Poisson- $\mu_{1}$ stream [10, Theorem 5.1]. For cycles with multiple bottlenecks, where the bottlenecks are now distributed over the cycle, the limiting picture is as follows.

Theorem 5.1. Let $\left(S_{1}^{(N)}, S_{2}^{(N)}, \ldots, S_{M}^{(N)}\right)$ denote a vector distributed according to $\phi^{(M, N)}\left(\theta_{1}\right.$, $\left.\ldots, \theta_{M}\right)$ from (2.3). Let $A:=\left\{l \in\{1, \ldots, M\}: \mu_{l} \neq \mu_{1}\right\}$ be the set of indices of the nonbottleneck nodes.

Then the sequence $\left(S_{j}^{(N)}, j \in A\right)$ converges for $N \rightarrow \infty$ to a vector with distribution

$$
\bigotimes_{j \in A} \exp \left(\mu_{j}-\mu_{1}\right)
$$

The proof is a direct consequence of (2.3) and the following theorem; see [17].

Theorem 5.2. The joint steady-state queue length distribution of the nonbottleneck nodes converges weakly to an independent product of geometrical distributions, which can be represented as the respective steady-state queue length distribution of an open ergodic tandem system with a Poisson- $\mu_{1}$ arrival stream, i.e. it holds that

$$
\mathrm{P}^{\left(X_{s}^{(M, N)}, s \in A\right)} \stackrel{\mathrm{D}}{\rightarrow} \bigotimes_{s \in A} \mathrm{Geo}\left(1-\frac{\mu_{1}}{\mu_{s}}\right) \quad \text { as } N \rightarrow \infty .
$$

\section{Central limit theorem for cycle times in a homogeneous cycle}

Our results in the previous sections are for cycles where at least two different service rates occur, i.e. we have bottleneck and nonbottleneck nodes. In this section we discuss the case in which all nodes have service intensity $\mu>0$. The first consequence is that the steady-state distribution is uniform on $Z(M, N)$. 
Theorem 6.1. In the homogeneous cycle the steady-state distribution is

$$
\pi^{(M, N)}\left(n_{1}, \ldots, n_{M}\right)=\left(\begin{array}{c}
M+N-1 \\
N
\end{array}\right)^{-1}, \quad\left(n_{1}, \ldots, n_{M}\right) \in Z(M, N) .
$$

Kelly [14, Corollary 3.2] proved a first-order limit for the cycle times in steady state.

Theorem 6.2. (Weak law of large numbers for the cycle time in homogeneous cycles.) Let $\mu_{1}=\cdots=\mu_{M}=1$. Then, for all $\varepsilon>0$,

$$
\mathrm{P}\left(\left|\frac{c_{N}}{N}-1\right| \geq \varepsilon\right) \leq\left(N+M^{2}\right) M^{-2} \varepsilon^{-2} \text { as } N \rightarrow \infty .
$$

Using the notation of the previous sections, we now formulate our main observation similarly to Theorem 3.3.

Theorem 6.3. (Central limit theorem for the cycle time in homogeneous cycles.) Let $\mu_{1}=$ $\cdots=\mu_{M}=: \mu$. Then

$$
\frac{c_{N}-\mathrm{E}\left[c_{N}\right]}{\sqrt{\operatorname{var}\left(c_{N}\right)}} \stackrel{\mathrm{D}}{\rightarrow} X \sim \mathcal{N}(0,1) \quad \text { as } N \rightarrow \infty
$$

Proof. From (2.2) and (6.1), we conclude that the cycle time has a gamma distribution, i.e. $c_{N} \sim \Gamma_{\mu, M+N-1}$, which is distributed as a sum of $M+N-1$ independent $\exp (\mu)$-distributed random variables.

Comparing this result with Theorem 3.3 we observe an obvious similarity. With regards to the central limit time and space scales, the result of Theorem 3.3 looks like the result of Theorem 6.3, i.e. as if all nonbottleneck nodes have been erased. However, comparing the proofs of the theorems reveals a delicate difference in the central limit theorems. Since in both theorems the accumulated mean $\mathrm{E}\left[c_{N}\right]$ and variance $\operatorname{var}\left[c_{N}\right]$ contribute to the standardization procedure, the nonbottleneck nodes do not disappear from the limiting procedure. On the other hand, from the viewpoint of the TC successively visiting the nodes of the cycle, the proof of Theorem 6.3 would rely on all individual sojourn times when $N \rightarrow \infty$, while the proof of Theorem 3.3 should eventually rely only on those individual sojourn times experienced by the TC at the bottlenecks.

In hindsight, we can interpret Theorem 6.3 by considering all nodes in the homogeneous cycle as bottlenecks.

\section{Cycle times in cycles with multiple-server nodes}

With multiple-server nodes, the problem of computing cycle time and sojourn time distributions becomes much more involved. This is visible even in the open tandem system of exponential multiple-server nodes.

In this situation, overtaking of customers comes into play, as was proved by Burke; see [5] and [6]. Excluding the case of infinite-server queues and summarizing briefly, the results are as follows. In steady state a customer's sojourn times on a passage through a tandem of exponential servers are independent as long as the interior nodes of the tandem are single servers. The first and the last nodes may each be a multiple server. The total passage time distribution is therefore the convolution of the sojourn time distributions. 
For closed systems, especially cycles, a similar theorem was proved under the heading of 'Burke's theorem on passage times in Gordon-Newell networks'. We investigate the random time between two entrances of the TC into node $Q[1]$, i.e. the cycle time of interest is characterized by starting at $Q[1]$.

We consider a cycle as in Section 2 with multiple servers at nodes 1 and $M$, having $s_{1}$ and $s_{M}$ service channels with service times $\mu_{1}$ and $\mu_{M}$, respectively. All other nodes are single servers, as before.

Proposition 7.1. The state space of the Markovian joint queue length process $X^{(M, N)}=$ $\left(X^{(M, N)}(t), t \geq 0\right)$ is $Z(M, N):=\left\{\left(n_{1}, \ldots, n_{M}\right) \in \mathbb{N}^{M} \mid n_{1}+\cdots+n_{M}=N\right\}$. The process $X^{(M, N)}$ is irreducible and positive recurrent. The limiting and stationary distribution is, with $\mu_{j}(m)=\mu_{j} \min \left(m, s_{j}\right)$ for $j=1, M$,

$$
\pi^{(M, N)}(n)=G(M, N)^{-1}\left(\prod_{m=1}^{n_{1}} \frac{1}{\mu_{1}(m)}\right) \prod_{i=2}^{M-1}\left(\frac{1}{\mu_{i}}\right)^{n_{i}}\left(\prod_{m=1}^{n_{M}} \frac{1}{\mu_{M}(m)}\right)
$$

with $n=\left(n_{1}, \ldots, n_{M}\right) \in Z(M, N)$ and normalising constant

$$
G(M, N)=\sum_{n \in Z(M, N)}\left(\prod_{m=1}^{n_{1}} \frac{1}{\mu_{1}(m)}\right) \prod_{i=2}^{M-1}\left(\frac{1}{\mu_{i}}\right)^{n_{i}}\left(\prod_{m=1}^{n_{M}} \frac{1}{\mu_{M}(m)}\right) .
$$

Theorem 7.1. (Limiting distribution of the cycle time and sojourn time vector [8].) The limiting distribution of the TC's cycle time is given by its LST

$$
\begin{aligned}
\psi^{(M, N)}(\theta)= & \sum_{n \in Z(M, N-1)} \pi^{(M, N-1)}(n) \prod_{j \in\{1, M\}}\left\{\frac{\mu_{j}}{\mu_{j}+\theta}\left(\frac{\mu_{j} s_{j}}{\mu_{j} s_{j}+\theta}\right)^{\left(n_{j}-s_{j}+1\right)_{+}}\right\} \\
& \times \prod_{j=2}^{M-1}\left(\frac{\mu_{j}}{\mu_{j}+\theta}\right)^{n_{j}+1}, \quad \theta \geq 0,
\end{aligned}
$$

where $\pi^{(M, N-1)}\left(n_{1}, \ldots, n_{M}\right)$ (defined in (7.1)) is the steady-state probability that at the arrival instants of the TC at node $Q[1]$, he observes $n_{1}$ customers before him at node $Q[1]$ and $n_{i}$ customers present at node $Q[i], i=2, \ldots, M$, without counting himself.

The limiting joint distribution of the TC's successive sojourn times during a cycle is of similar structure; see [16]. If we define the cycle time for the TC to be the time between two successive entrances of the TC into some other node $Q[i], i \neq 1$, the cycle time distribution would change. Furthermore, (7.2) no longer holds [8, p. 880-881] and, to date, the cycle time distribution seems to be unknown.

With respect to the investigation in this paper we conclude that it matters whether there is at, say, node $2<M$ one server with service rate $\mu_{2}$ or two servers with service rate $\mu_{2} / 2$. The complete picture of the mode of convergence of the cycle time distribution when the population size $N$ increases unboundedly is part of our ongoing research, but is still an open problem.

\section{Acknowledgement}

We thank the anonymous referee for his/her careful reading of the manuscript and constructive criticism: Sections 6 and 7 emerged from his/her comments. 


\section{References}

[1] Bickel, P. J. and Doksum, K. A. (1977). Mathematical Statistics: Basic Ideas and Selected Topics. PrenticeHall.

[2] Boxma, O. J. (1983). The cyclic queue with one general and one exponential server. Adv. Appl. Prob. 15, 857-873.

[3] Boxma, O. J. (1988). Sojourn times in cyclic queues - the influence of the slowest server. In Computer Performance and Reliability, eds G. Iazeolla, P.-J. Courtois and O. J. Boxma. North-Holland, Amsterdam, pp. 13-24.

[4] Boxma, O. J., Kelly, F. P. and Konheim, A. G. (1984). The product form for sojourn time distributions in cyclic exponential queues. J. Assoc. Comput. Mach. 31, 128-133.

[5] Burke, P. J. (1968). The output process of a stationary M/M/s queueing system. Ann. Math. Statist. 39, 1144-1152.

[6] Burke, P. J. (1969). The dependence of sojourn times in tandem $M / M / s$ queues. Operat. Res. 17, 754-755.

[7] Chow, W. M. (1980). The cycle time distribution of exponential cyclic queues. J. Assoc. Comput. Mach. 27, 281-286.

[8] Daduna, H. (1984). Burke's theorem on passage times in Gordon-Newell networks. Adv. Appl. Prob. 16, 867-886.

[9] Daduna, H. and SzeKLI, R. (2002). Conditional job-observer property for multitype closed queueing networks. J. Appl. Prob. 39, 865-881.

[10] Daduna, H., Malchin, C. And Szekli, R. (2008). Weak convergence limits for sojourn times in cyclic queues under heavy traffic conditions. J. Appl. Prob. 45, 333-346.

[11] Gordon, W. J. and Newell, G. F. (1967). Closed queueing networks with exponential servers. Operat. Res. 15, 254-265.

[12] Harrison, P. G. (1985). On normalizing constants in queueing networks. Operat. Res. 33, 464-468.

[13] Harrison, P. G. (1990). Laplace transform inversion and passage-time distributions in Markov processes. J. Appl. Prob. 27, 74-87.

[14] Kelly, F. P. (1984). The dependence of sojourn times in closed queueing networks. In Mathematical Computer Performance and Reliability, eds G. Iazeolla, P.-J. Courtois and A. Hordijk, North-Holland, Amsterdam, pp. 111121.

[15] Malchin, C. (2008). Stochastic networks: discrete and continuous time models. Doctoral Thesis, Department of Mathematics, University of Hamburg.

[16] Schassberger, R. And Daduna, H. (1987). Sojourn times in queuing networks with multiserver nodes. J. Appl. Prob. 24, 511-521.

[17] Stenzel, O. and Daduna, H. (2009). Weak convergence limits for closed cyclic networks of queues with multiple bottleneck nodes. Preprint 2009-05, Mathematische Statistik und Stochastische Prozesse, Fachbereich Mathematik der Universität Hamburg, 22 pp.

[18] Serfozo, R. F. (1999). Introduction to Stochastic Networks (Appl. Math. 44). Springer, New York. 